\title{
Modelling multiscale collective behavior with Gaussian processes
}

\author{
Nazareno Campioni ${ }^{1}$, Dirk Husmeier ${ }^{1}$, Juan M. Morales ${ }^{2}$, Jennifer Gaskell ${ }^{1}$ and Colin J. Torney $^{1}$ \\ ${ }^{1}$ School of Mathematics and Statistics, University of Glasgow, UK \\ ${ }^{2}$ Grupo de Ecología Cuantitativa, INIBIOMA-CONICET, Universidad Nacional del Comahue, Argentina
}

\begin{abstract}
Collective behavior is characterized by the emergence of large-scale phenomena from local interactions. It is found in many contexts, including political movements, fads and fashions, and animal grouping. In this paper, we aim to elucidate the mechanisms that underlie observed collective behavior by developing a novel mathematical framework based on equation-free modelling procedures and Gaussian process regression. This allows us to circumvent the possible lack of formal mathematical links between scales and instead use statistical emulation to learn an empirical Fokker-Planck equation. Our approach advances our ability to understand how complex systems function at both the individual and collective level when a formal mathematical description of macroscale dynamics is unavailable.
\end{abstract}

Keywords: Collective behavior, Empirical Fokker-Planck equation, Equation-free modelling, Statistical emulation.

\section{Introduction}

Complex systems are characterized by interacting components that give rise to emergent collective behavior. Examples of complex systems can be found in domains such as ecology, neuroscience, economics and politics. Understanding how these systems function requires an approach that combines individual behaviour, the nature of interactions, and the group-level phenomena that arise. In the context of animal movement, inferring the dynamics and structural properties of these systems has largely focused on the micro-scale [1, 2], using random walk models [3, 4], step selection functions [5, 6] or analysis of movement correlations [7]. A frequently encountered issue when developing statistical or mechanistic models of interacting systems is that a model that captures observed phenomena at one level, for example the individual-level, does not match or predict observations of the same system at another level, for example the group-level. In fact, for collective animal movement it has been shown that fitting models to fine scale interaction dynamics, such as individual turn rates, leads to inconsistencies with models developed to match macro-scale dynamics, such as group alignment [8]. Linking individual and group-scale phenomena can be done through equation-free modelling, where the goal is to approximate the evolution of the system at a macroscopic, coarse scale, while mechanistic models are only given at a more detailed, fine scale. However, the explicit derivation of the coarse-scale equations is often intractable, and they might not have any analytically closed form.

The idea of equation-free modelling is to pursue a surrogate approach based on three steps: lifting, simulation, and restriction (see [9] for more details). In this paper, we propose to bypass the need for a formal mathematical link between multiple scales and develop a statistical method analogous to equation-free modelling. The procedure involves statistical emulation of an unknown coarse-grained description of the system via massively parallel simulations of micro-scale models. On the other hand, the macro states are equation free since a mathematical description, which exists in principle, is usually unknown in practice. Previous work has pursued a parametric route, trying to fit, typically heuristically, the parameters of an assumed model, through means of an empirical Fokker-Planck equation (Eq2), which is characterized by an average velocity-dependent diffusion and drift term [10]. However, this usually leads to a bias, in that the parametric model deviates from the unknown, true macro dynamics. To avoid the bias, we integrate the micro dynamics with a semi-parametric approach, using a statistical emulator based on a Gaussian process prior [11]. To this end, the fine-scale equations are numerically solved in parallel to provide a set of solutions that are local in space, time and parameter domain. The emulator will then target the drift and diffusion term as functions of some macroscopic variables (defined in the next section), leading to what effectively is a latent Gaussian process entering the empirical dynamical equations.

\section{Methods and Results}

To develop our framework, we borrow the mathematical model which describes the micro-scale interactions between desert locusts (Schistocerca gregaria) from [12]. In the experimental component of Buhl et al. [12], locusts are placed in a ring-shaped arena and their motion is recorded for 8 hours. The empirical data is then compared to a model that describes the 
locusts' behaviour through the following modified, 1-dimensional self-propelled particles (SPPs) model:

$$
x_{i}(t+1)=x_{i}(t)+v_{0} u_{i}(t), \quad u_{i}(t+1)=\alpha u_{i}(t)+(1-\alpha) G\left(\langle u(t)\rangle_{i}\right)+\xi_{i},
$$

with $x_{i}$ and $u_{i}$ being, respectively, a single locust's position and velocity. The quantity $\langle u(t)\rangle_{i}$ represents the average velocity of all locusts, excluding locust $i$, within the interaction range $\left[x_{i}-\Delta, x_{i}+\Delta\right]$ and $G$ is a function which represents the adjustment of a particle velocity to the velocity of its neighbors: $G(x)= \begin{cases}(x+1) / 2, & x>0 \\ (x-1) / 2, & x<0 .\end{cases}$

We omit further details and invite the reader to consult [12] for a thorough explanation. Thus, locusts behave as particles that change their velocity in response to near neighbors, which makes it possible to produce those sudden, random changes in directions that are evidence of the intrinsic stochasticity of animal groups movements. Our parameters of interest are the locusts' interaction range $\Delta$, the white noise term $\xi_{i}$, which is randomly chosen with uniform probability from the interval $\left[-\frac{\eta}{2}, \frac{\eta}{2}\right]$, and $\alpha$, the relative weight that a locust assigns to its own velocity and that of its neighbors in deciding its own velocity [12]. Critical value $\alpha=1$ represents the dynamics of one locust in absence of any other individual.

We wish to analyze the micro-scale model through means of a macro-scale quantity which can describe the group dynamics (this is referred to as restriction in the equation-free modelling approach). Such a quantity is the global average velocity $U$. This choice is justified by the fact that the model from [12] is built in such a way that $U$ values close to 1 or -1 indicate a state of alignment in the marching locusts (specifically, by the convention we have adopted, positive values correspond to movements to the right and negative values correspond to movements to the left). In line with [10], we assume that in the case of finite interaction radius $\Delta$, an empirical Fokker-Planck equation (FPE) (Eq 2) exists and approximations of its solution can be found. Let $f(U, t)$ be the probability density function of $U$ and let $F(U)$ and $D(U)$ represent the drift and diffusion function, which give a quantification of, respectively, the mean rate of change and the randomness of the evolution of $U$. Then $f(U, t), F(U)$ and $D(U)$ are related via the following equation:

$$
\frac{\partial f}{\partial t}=\frac{\partial^{2}(D(U) f)}{\partial U^{2}}-\frac{\partial(F(U) f)}{\partial U}
$$

In particular, we focus on the stationary probability density (SPD), $\frac{\partial f}{\partial t}=0$.

In order to estimate $F$ and $D$, Yates et al. [10] performed a large number of short time simulations taking an ensemble average of $\mathrm{Eq} 3$ as approximations to the drift and diffusion function.

$$
F(U)=\frac{U(t+\delta t)-U(t)}{\delta t}, \quad D(U)=\frac{1}{2} \frac{(U(t+\delta t)-U(t))^{2}}{\delta t}
$$

They calculated these two quantities with different time steps as they discovered that smaller $\delta t$ values yield better approximations to $D(U)$ whereas better approximations to $F(U)$ are produced by larger $\delta t$ values.

We will now explain our main innovation which substantially differs from the work of Yates et al. [10]. The objective is to use Gaussian process regression to find estimates for $D(U)$ and $F(U)$. In Gaussian process regression settings, a dependent variable $y_{i}$ is modelled through independent variables $x_{i}$ via $y_{i}=f\left(x_{i}\right)+\epsilon_{i}$, where $\epsilon_{i}$ are independent, Gaussian noise and $f$ is a random object seen as the realization of a Gaussian process. Gaussian processes are completely determined by a mean function $m(x)$, which we set to 0 throughout this paper [13], and a kernel function $K\left(x_{1}, x_{2}\right)$. Applying GP regression to the drift and diffusion inference then simplifies to solving a regression problem where the dependent variable and the noise variance are given by $\mathrm{Eq} 4$

$$
y_{i}=\frac{U\left(t_{i}+\delta t\right)-U\left(t_{i}\right)}{\delta t}, \quad \sigma_{i}^{2}=\frac{D\left(U\left(t_{i}\right)\right)}{\delta t}
$$

In line with [13], the diffusion function is modelled with an RBF kernel and we use exponential and sigmoid transformations to ensure positiveness of the lenghtscale parameters. On the other hand, the drift function is fit to another Gaussian process where the diffusion posterior mean is used as the observation noise variance ( $\sigma_{i}^{2}$ in Eq 4 ). By using TensorFlow, we run 1000 
simulations in parallel for 50000 time steps, disregarding the first 250 values (the burn-in phase). The parameter values used are $\alpha=0.66, \Delta=2$ and $\eta=0.8$. For each simulation, 20 individuals are initialized at different positions and updated at a single time step, although only individuals within the same simulation interact. We then take the average velocity of each simulation and use these values as inputs to Eq 3 Time step $\delta t=1$ is kept equal for both quantities. We can also draw random samples from the posterior distribution of the GPs, so as to obtain an uncertainty quantification around the posterior mean. This is shown if Fig.1. which shows a bimodal double-well profile for the diffusion function (left panel), and a sinusoidal pattern of the drift function (right panel).
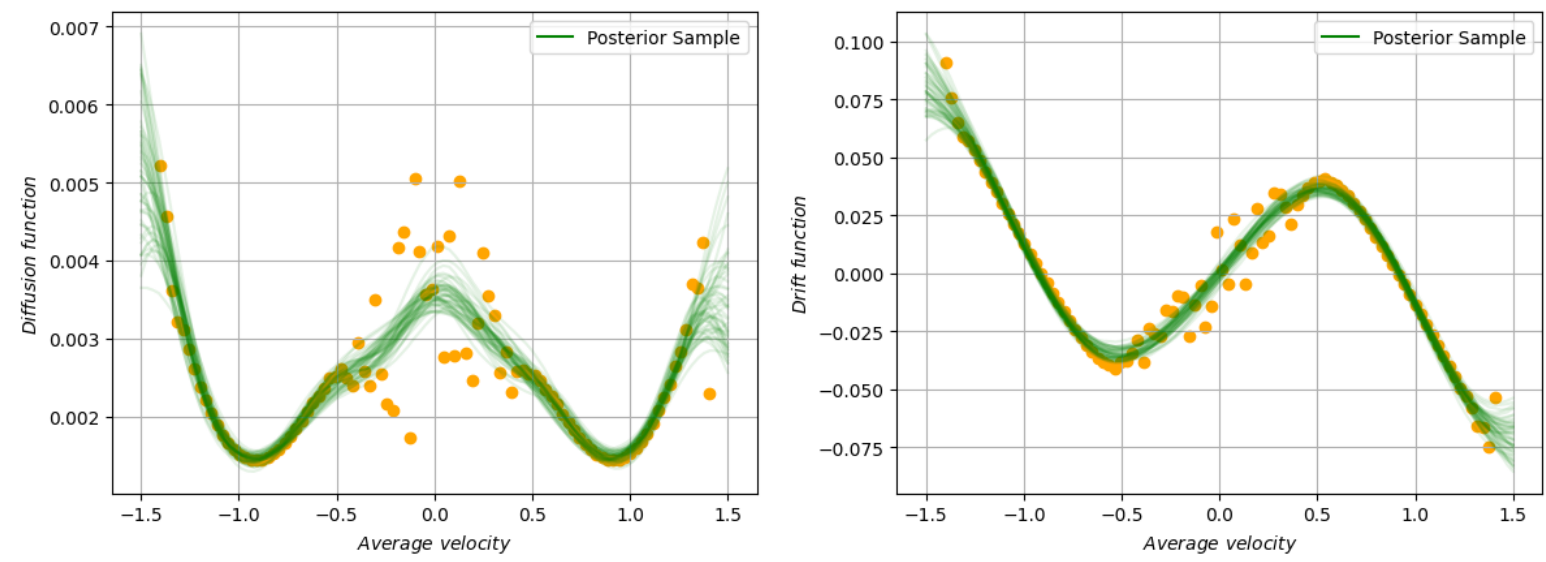

Fig. 1: GP samples for diffusion and drift function $D(U), F(U)$. Orange dots represent the observed points.

As [10] suggests, the SPD can be obtained via

$$
f(U)=C \exp (-\phi(U)) \quad(S P D), \quad \phi(U)=-\int_{0}^{U} \frac{F(s)}{D(s)} d s-\ln (D(U)) \quad(\text { potential }) .
$$

Taking random samples from the drift and diffusion distribution allows us to straightforwardly propagate the uncertainty through Eq.5. We show our results in Fig.2. In the latter, we include a histogram of the locusts' average velocity from our TensorFlow simulation (code available upon request).
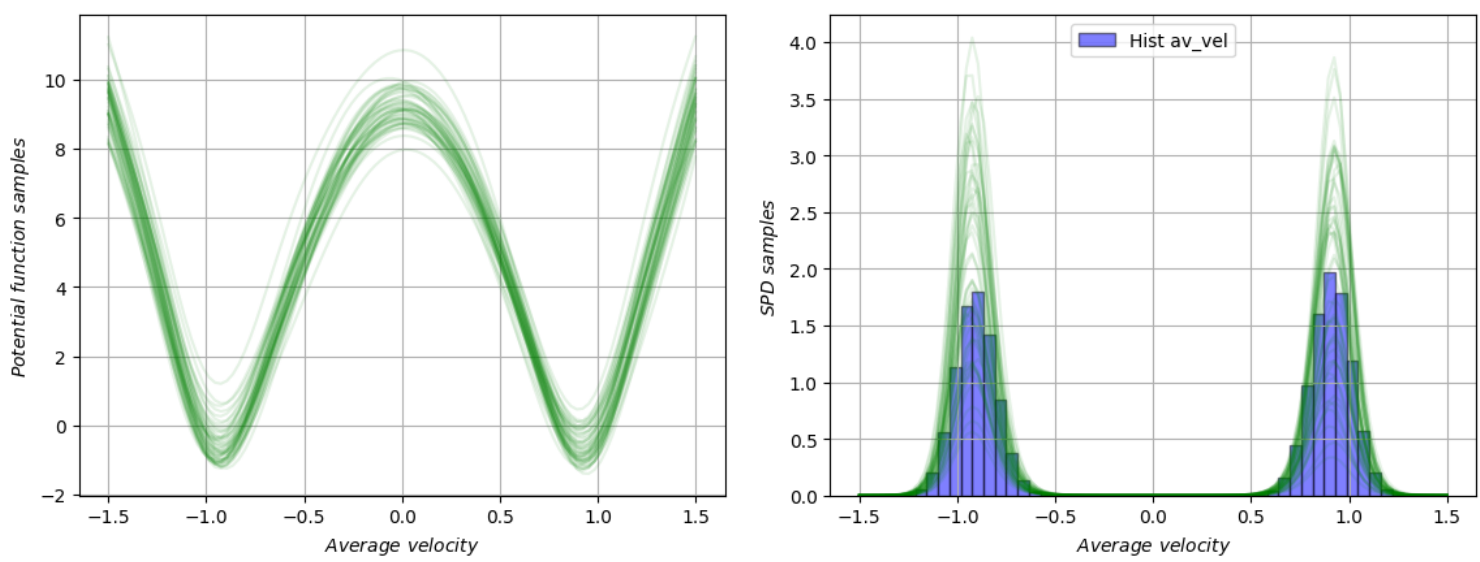

Fig. 2: Potential and SPD samples given by Eq 5 , average velocity histogram and GP samples from the posterior distribution.

\section{Conclusion}

In this paper, we have developed a new method which bypasses the need of a formal, mathematical description of the link between micro and macro-scale dynamics of collective movement. We have borrowed ideas from already existing methods in equation-free modelling and approximate Bayesian learning of stochastic differential equations and combined them into 
a general framework to model collective behavior through Gaussian processes. Assuming the existence of an empirical Fokker-Planck equation governing the evolution of the group average velocity [10] and incorporating equation-free modelling principles with a statistical emulator [13] resulted into an efficient methodology for collective behavior predictions, which are in good agreement with our simulations. The resulting stationary probability density shows bi-modality, main characteristic of the locusts' dynamics: the group will tend to move toward the same direction, represented by average velocity values close to 1 and -1 , whereas chaotic, disordered movement, represented by average velocity values close to 0 , is not a feature of this collective movement.

Although our framework can be applied to different instances of collective movement which can be found in various disciplines, such as ecology, politics or economics, we have chosen as our toy model a simple one-dimensional model representing the motion of desert locusts. Hence, there are different directions that future projects can take. We are currently working on developing a new inferential framework for inferring micro-scale dynamics from static measurements of interacting systems by combining standard MCMC algorithms with statistical emulations. In particular, we are aiming to integrate local approximations of the log-likelihood of the locusts' system into Metropolis-Hastings kernels using Gaussian process regression [14]. Other routes which will be pursued include extending our ideas to two-dimensional models and applying our new procedures to real data, for example data of wildebeest in the Serengeti national park in Tanzania or data of sheep roaming in Patagonia.

\section{References}

[1] T. Mora, A. Walczak, L. Del Castello, F. Ginelli, S. Melillo, L. Parisi, M. Viale, A. Cavagna, and I. Giardina, "Local equilibrium in bird flocks," Nature Physics, vol. 12, p. 1153-1157, 2016.

[2] M. B. Hooten, H. R. Scharf, T. J. Hefley, A. T. Pearse, and M. D. Weegman, "Animal movement models for migratory individuals and groups," Methods Ecol Evol, vol. 9, no. 7, pp. 1692-1705, 2018.

[3] C. J. Torney, J. G. C. Hopcraft, T. A. Morrison, I. D. Couzin, and S. A. Levin, "From single steps to mass migration: the problem of scale in the movement ecology of the serengeti wildebeest," Philos T Roy Soc B, vol. 373, no. 1746, p. 20170012, 2018.

[4] T. Patterson, A. Parton, R. Langrock, P. Blackwell, L. Thomas, and R. King, "Statistical modelling of individual animal movement: an overview of key methods and a discussion of practical challenges," ASTA-Adv Stat Anal, 072017.

[5] J. R. Potts, K. Mokross, and M. A. Lewis, "A unifying framework for quantifying the nature of animal interactions," Journal of The Royal Society Interface, vol. 11, no. 96, p. 20140333, 2014.

[6] M. del Mar Delgado, V. Penteriani, J. Manuel Morales, E. Gurarie, and O. Ovaskainen, "A statistical framework for inferring the influence of conspecifics on movement behaviour," Methods Ecol Evol, vol. 5, no. 2, pp. 183-189, 2014.

[7] M. Nagy, Z. Ákos, D. Biro, and T. Vicsek, "Hierarchical group dynamics in pigeon flocks," Nature, vol. 464, pp. 890-893, 2010.

[8] R. P. Mann, A. Perna, D. Strömbom, R. Garnett, J. E. Herbert-Read, D. J. T. Sumpter, and A. J. W. Ward, "Multi-scale Inference of Interaction Rules in Animal Groups Using Bayesian Model Selection," PLoS Comp Bio, vol. 8, pp. 1-12, 012012.

[9] I. G. Kevrekidis and G. Samaey, "Equation-Free Multiscale Computation: Algorithms and Applications," Annual Review of Physical Chemistry, vol. 60, no. 1, pp. 321-344, 2009.

[10] C. A. Yates, R. Erban, C. Escudero, I. D. Couzin, J. Buhl, I. G. Kevrekidis, P. K. Maini, and D. J. T. Sumpter, "Inherent noise can facilitate coherence in collective swarm motion," PNAS, vol. 106, no. 14, pp. 5464-5469, 2009.

[11] S. Roberts, M. Osborne, M. Ebden, S. Reece, N. Gibson, and S. Aigrain, "Gaussian processes for time-series modelling," Philos T Roy Soc A, vol. 371, no. 1984, p. 20110550, 2013.

[12] J. Buhl, D. J. T. Sumpter, I. D. Couzin, J. J. Hale, E. Despland, E. R. Miller, and S. J. Simpson, "From Disorder to Order in marching locusts," Science, vol. 312, no. 5778, pp. 1402-1406, 2006.

[13] P. Batz, A. Ruttor, and M. Opper, "Approximate Bayes learning of stochastic differential equations," Physical Review E, vol. 98, no. 2, p. 022109, 2018.

[14] P. R. Conrad, Y. M. Marzouk, N. S. Pillai, and A. Smith, "Accelerating asymptotically exact MCMC for computationally intensive models via local approximations," J Am Stat Assoc, vol. 111, no. 516, pp. 1591-1607, 2016. 